\title{
Fluorescence imaging in hepatobiliary surgery - hope and hype
}

\author{
Shridhar Ganpathi lyer $^{1,2,3}$, FRCS, MBA
}

S urgery is constantly evolving, with innovations in surgical techniques and minimally invasive, endoscopic and robotic surgery. Most surgical procedures rely on white-light reflectance, which does not allow differentiation between normal and diseased tissue beyond gross anatomical appearances. Fluorescence imaging ( $\mathrm{Fl}$ ) offers enhancement to traditional low-contrast white-light visualisation and real-time, pseudocolour delineation of anatomic structures. ${ }^{(1)}$ The use of $\mathrm{Fl}$ for intraoperative navigation provides the surgeon with enhanced visualisation capabilities, including localisation of anatomical structures; delineation of diseased and normal tissue; enhanced localisation of tumours and lymph nodes; intraoperative assessment of margin status; and real-time visualisation of organ perfusion, all of which can deliver better outcomes. To obtain a fluorescence signal, the operative field is exposed to nearinfrared (NIR) light sources, while the target is injected with a fluorescent dye or contrast agent that emits a fluorescence signal after being excited by laser sources. The fluorescence signal can be either visualised directly on the operative field during surgical procedures or captured by specific cameras and displayed on a screen in a minimally invasive procedure or endoscopy. ${ }^{(2)}$ In this way, FI allows surgical navigation without the need for additional bulky equipment in the operating room, and the enhanced information provided to the surgeon is displayed in real time.

Several targeted contrast agents are established in clinical use and a number of agents are under development. The most widely used agents include indocyanine green (ICG), fluorescein, methylene blue and 5-aminolevulinic acid. A large number of contrast agents that are in development are small moleculebased, followed closely by peptide- or antibody-based contrast agents and nanoparticles. ${ }^{(3)} \mathrm{A}$ wide array of clinically approved $\mathrm{Fl}$ systems are available for open surgery, minimally invasive and endoscopic procedures.

FI that utilises the fluorescent properties of ICG fluorophores (most commonly used in FI) is increasingly being adopted in various medical and surgical disciplines. ICG is a watersoluble, tricarbocyanine dye that binds to blood lipoproteins after intravenous injection. ICG is selectively taken up at the first pass by hepatocytes and excreted unchanged into the bile. This fluorophore has tissue penetration of up to $5 \mathrm{~mm}$, a plasma half-life of 3-5 minutes and biliary excretion time of 15-20 minutes, making it ideal for repeated applications. ${ }^{(2)}$ The plethora of publications related to $\mathrm{FI}$ and guided surgery is a testament to the increased adoption and interest in the field. ${ }^{(2)}$
ICG FI is currently used in multiple disciplines in medical fields. ICG angiography in neurosurgery has become a routine method for intraoperative evaluation of intracranial blood circulation in aneurysm surgery ${ }^{(4)}$ and the patency of microanastomoses in neurosurgical extracranial-intracranial revascularisation operations. ${ }^{(5)}$ In cardiovascular surgery, ICG FI is used for intraoperative assessment of graft patency, diagnostics of peripheral arterial occlusive disease and predicting wound healing after major amputation. ${ }^{(6)}$ In oncologic surgery, ICG FI has been found to be useful for mapping lymphatics and nodes in breast, gastric, colorectal, gynaecologic, and head and neck cancers. ${ }^{(7)}$ In colorectal surgery, $\mathrm{Fl}$ is found to be useful in assessing anastomotic perfusion, ${ }^{(8)}$ visualisation of the ureter, endoscopic tattooing of lesions, detection of small carcinomatosis and liver metastasis. ${ }^{(9)}$

Specifically in the arena of hepatobiliary and pancreatic surgery, ICG FI has several applications. The exclusively hepatic metabolism of ICG, which results in biliary excretion starting approximately 30 minutes after intravenous injection, enables visualisation of the biliary anatomy, which is useful during difficult cholecystectomies. The advantages of ICG FI over conventional radiographic cholangiography are convenience, time savings, the ability to delineate the anatomy in real time before the dissection of Calot's triangle, and safety, although it is not useful in detecting stones. The FALCON trial, ${ }^{(10)}$ a multicentre randomised controlled trial, is currently investigating the use of ICG FI in laparoscopic cholecystectomies. The trial is powered with the primary endpoint of time to 'critical view of safety' and not bile duct injury, as the latter requires a much larger sample size and may be impractical. In liver surgery for clear identification of segmental liver anatomy, Ishizawa et al has demonstrated positive or negative staining techniques for liver segment delineation in laparoscopic hepatic resections. ${ }^{(11)}$ During positive segmental staining, the portal vein is punctured under sonographic guidance through the liver surface with slow injection of ICG. In the negative staining technique, the Glissonean pedicle that corresponds to the segments to be resected is dissected and clamped, followed by the use of intravenous ICG, which stains the remaining liver. ICG clearance is impaired in malignant hepatocytes or liver tissue that is compressed by a tumour, a property that may aid in tumour detection if ICG is injected preoperatively. However, it is only useful for subcapsular lesions, as the depth of penetrance is limited to $8 \mathrm{~mm}$ for NIR imaging. Another limitation is the high incidence of false positives despite the high sensitivity. ${ }^{(12)}$

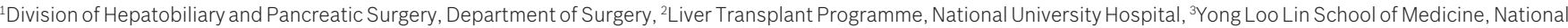
University of Singapore, Singapore

Correspondence: Dr Shridhar Ganpathi lyer, Senior Consultant and Head, Division of Hepatobiliary and Pancreatic Surgery, Department of Surgery, National University Hospital, Singapore119228.surisg@nus.edu.sg 
Another application of ICG FI is in securing surgical margins and modifying the plane of transection if fluorescence is encountered. ${ }^{(13)}$ Newton et al has also described the use of ICG $\mathrm{Fl}$ in identifying tumours and real-time assessment of pancreatic margins in pancreatic resections. ${ }^{(14)}$

ICG FI still has several limitations, such as the lack of quantitative data to measure tissue perfusion (i.e. 'How green is green?') and the lack of standardised and validated quantitative models. In this issue of the Singapore Medical Journal, Lim et al ${ }^{(15)}$ described their experience with NIR imaging using ICG for intraoperative liver tumour localisation in 32 patients. A total of 46 lesions were resected, including 23 hepatocellular carcinomas, 12 colorectal liver metastases and 11 benign lesions. ICG Fl identified 38 (82.6\%) out of 46 lesions prior to resection. For the eight lesions not detected by ICG, 6 (75.0\%) out of eight were in cirrhotic livers, and 1 (12.5\%) was $>1 \mathrm{~cm}$ deep. Lim et al also observed that in cirrhotic patients with malignant lesions, those given ICG on preoperative Days 4-7 had a higher detection rate of $91.7 \%$, compared to $66.7 \%$ if it was administered on Days 0-3. Based on these observations, they concluded that it is a useful modality and that the injection of ICG can be done nearer to the resection day provided that the liver is not cirrhotic. Further areas to explore in this study would be the concordance among the preoperative imaging or localisation, intraoperative detection with ICG and postoperative pathology report, as well as the number of patients who had a revision of surgical plan based on intraoperative FI.

Despite the initial promise of $\mathrm{FI}$ in various surgical fields, enthusiasm has outstripped the evidence. Adoption of newer techniques or technology even before efficacy is established may lead to increased costs and possibly harm. However, given the ease of application of $\mathrm{FI}$ and the relative safety of the procedure, it is likely that FI ICG will be adopted widely prior to systematic attempts at seeking evidence, even if the benefit is marginal compared to existing modalities. The only barrier to adoption is the availability of systems and hospitals that invest in the additional costs. For instance, the da Vinci® (Intuitive Surgical Inc, CA, USA) robotic system incorporates NIR capabilities and using ICG will incur only the cost of the drug. Future systems for minimally invasive surgery will also incorporate NIR capabilities. In addition to factors such as compatibility with current practice, the surgeons' learning curve, and demand from patients or surgeons, the major driver for change is economic: the NIR imaging market is projected to reach USD 822 million by 2023 from an estimated USD 416 million in 2018, a compound annual growth rate of $14.6 \% .{ }^{(16)}$
Finally, even if the retrospective data emerges, some questions remain, as is true whenever there is rapid adoption of new technology in clinical practice. We need to consider if it is possible to conduct randomised controlled trials with FI ICG to seek evidence and whether it remains justifiable to offer traditional care to patients, given that our medical ethics call for the benefits of new or experimental treatments to be distributed equally among all groups in society. Only then will this technology offer more hope than hype to patients.

\section{REFERENCES}

1. Orosco RK, Tsien RY, Nguyen QT. Fluorescence imaging in surgery. IEEE Rev Biomed Eng 2013; 6:178-87.

2. Alander JT, Kaartinen I, Laakso A, et al. A review of indocyanine green fluorescent imaging in surgery. Int J Biomed Imaging 2012; 2012:940585.

3. Barth CW, Gibbs SL. Fluorescence image-guided surgery - a perspective on contrast agent development. Proc SPIE Int Soc Opt Eng 2020; 11222:112220J.

4. Raabe A, Nakaji P, Beck J, et al. Prospective evaluation of surgical microscopeintegrated intraoperative near-infrared indocyanine green videoangiography during aneurysm surgery. J Neurosurg 2005; 103:982-9.

5. Woitzik J, Horn P, Vajkoczy P, Schmiedek P. Intraoperative control of extracranial-intracranial bypass patency by near-infrared indocyanine green videoangiography. J Neurosurg 2005; 102:692-8.

6. Desai ND, Miwa S, Kodama D, et al. Improving the quality of coronary bypass surgery with intraoperative angiography: validation of a new technique. J Am Coll Cardiol 2005; 46:1521-5

7. Zeng HC, Hu JL, Bai JW, Zhang GJ. Detection of sentinel lymph nodes with near-infrared imaging in malignancies. Mol Imaging Biol 2019; 21:219-27.

8. Jafari MD, Wexner SD, Martz JE, et al. Perfusion assessment in laparoscopic left-sided/anterior resection (PILLAR II): a multi-institutional study. J Am Coll Surg 2015; 220:82-92.e1.

9. Gossedge G, Vallance A, Jayne D. Diverse applications for near infra-red intraoperative imaging. Colorectal Dis 2015; 17 Suppl 3:7-11.

10. van den Bos J, Schols RM, Luyer MD, et al. Near-infrared fluorescence cholangiography assisted laparoscopic cholecystectomy versus conventional laparoscopic cholecystectomy (FALCON trial): study protocol for a multicentre randomised controlled trial. BMJ Open 2016; 6:e011668.

11. Ishizawa T, Zuker NB, Kokudo N, Gayet B. Positive and negative staining of hepatic segments by use of fluorescent imaging techniques during laparoscopic hepatectomy. Arch Surg 2012; 147:393-4.

12. Ishizawa T, Fukushima N, Shibahara J, et al. Real-time identification of liver cancers by using indocyanine green fluorescent imaging. Cancer 2009; 115:2491-504.

13. Aoki T, Murakami M, Koizumi T, et al. Determination of the surgical margin in laparoscopic liver resections using infrared indocyanine green fluorescence. Langenbecks Arch Surg 2018; 403:671-80.

14. Newton AD, Predina JD, Shin MH, et al. Intraoperative near-infrared imaging can identify neoplasms and aid in real-time margin assessment during pancreatic resection. Ann Surg 2019; 270:12-20.

15. Lim HJ, Chiow AKH, Lee LS, et al. Novel method of intraoperative liver tumour localisation with indocyanine green and near-infrared imaging. Singapore Med J $2021 ; 62: 182-9$.

16. Markets and Markets. Near Infrared Imaging Market by Product Type (NIR Fluorescence Imaging, Reagents), Application (Cancer surgery, Plastic surgery, Gastrointestinal surgery) End Users (Hospitals \& Clinics, Research laboratory, Pharmaceutical companies) - Global Forecast to 2023. Available at: https://www.marketsandmarkets.com/Market-Reports/near-infrared-imagingmarket-174869738.html. Accessed February 27, 2021 